\section{RANOVA: A FORTRAN IV program for four-way Monte Carlo analysis of variance}

\section{A. P. WHITE, A. W. STILL, and P. W. HARRIS Durham University, Durham, England}

Problems arise when it is necessary to perform an analysis of variance on data that do not show normally distributed within-cells error variance, one of the assumptions on which the conventional analysis is based. Traditional nonparametric tests exist only for a few simple designs. But a transformation to "normalize" the data prior to using the conventional parametric procedure can cause distortion of ordinal interactions and, as Myers (1966) has illustrated, can lead to nonmonotonic transformations of the means.

Another approach is to carry out an approximate randomization test for each $\mathrm{F}$ ratio, in the manner described by Edgington (1969). This involves using the $F$ ratio as a nonparametric test statistic within a framework that is best described as one that is isomorphic with the corresponding parametric model. This is the approach adopted in this program.

Description. The program is capable of dealing with any fixed-effects analysis of variance design with up to four factors and a maximum of 20,000 scores. $^{1}$ It evaluates the significance level of each $F$ ratio separately by subtracting all the effects from the data (except the one under consideration) and taking a random sample (with replacement) of the permissible data arrangements. In the case of between-subjects factors, the data arrangements comprise all between-subjects permutations; that is, the cells in the design function as the units of permutation. For within-subjects factors, on the other hand, the data arrangements comprise all within-subjects permutations; that is, each subject's scores are permuted separately. The $\mathrm{F}$ ratio is computed for each permuta-

This work was financed by the Manpower Services Commission as Job Creation Project N/671/76. tion and a cumulative probability distribution is thus produced for each individual $F$ ratio. The significance level of each $F$ ratio is then calculated by computing the ordinal position of the $\mathrm{F}$ ratio in the distribution concerned. Edgington (1969) gives a full account of this part of the procedure.

Computer and Language. RANOVA is written in FORTRAN IV with an assembler-written subroutine for providing dynamic storage allocation on the IBM $370 / 168$.

Restrictions. The storage capacity of the program is at present limited to 20,000 scores. However, this can be changed by altering a single statement that is indicated at the beginning of the listing.

Core Requirements. The program requires approximately 200,000 bytes.

Time Requirements. Time requirements can be heavy, especially for the more complex analysis of variance designs and for cases with large numbers of scores. The time taken also depends on the precision with which the randomization probabilities are required. Comprehensive guidelines are given in the manual. However, a four-way design, with two levels of each factor and 10 scores per cell, takes about $130 \mathrm{sec}$ with the number of permutations for each effect set at 100 .

Availability. A manual and listing are available without cost from A. P. White, Department of Psychology, Durham University, Science Laboratories, South Road, Durham DH1 3LE England.

\section{REFERENCES}

EDgington, E. A. Approximate randomization tests. Jourmal of Psychology, 1969, 72, 143-149.

MYERs, J. L. Fundamentals of experimental design. Boston: Allyn \& Bacon, 1966.

\section{NOTE}

1. I am grateful to $R$. Sheehan, University of Durham Computer Unit, for providing an assembler-written subroutine for achieving dynamic storage allocation.

(Accepted for publication June 16, 1978.) 